\title{
New and Emerging Mobile Technologies for Health Care (mHealth): A Horizon Scanning Review
}

\author{
A Kazemi ${ }^{1,} ;$ H Salmani ${ }^{2}$; A Shakibafard ${ }^{3}$; F Fatehi ${ }^{4}$ \\ 1 Shiraz University of Medical Sciences, Shiraz, Iran \\ ${ }^{2}$ School of Health Management and Information Sciences, Iran University of Medical Sciences, Tehran, Iran \\ 3. Shiraz University of Medical Sciences, Shiraz, Iran \\ ${ }^{4}$ School of Allied Medical Sciences, Tehran University of Medical Sciences, Tehran, Iran \\ * Corresponding author: A Kazemi, MSc of Medical Informatics, Information Technology Department, School of Paramedical, Shiraz University of Medical Sciences, Shiraz, Iran. \\ Tel: +98-7132270240, E-mail: azarKazemi.mi@gmail.com
}

Received: 11 Dec 2016

Accepted: 01 Jan 2017

Epub: 23 Feb 2017

Ppub: 15 Jan 2018

\begin{abstract}
Background: Mobile technologies deliver new capabilities that can be beneficial to drive major aspects of health care. In patientcentered healthcare there is a need for continuing monitoring health technologies to enable the fast and accurate decision making.

Objectives: The aim of this study was to explore and identify new and emerging technologies that are likely to impact the future of health care and practical research.

Methods:We conducted a systematic search on top ranking health technology websites according to Alexa Rank. The websites included msn.com, theguardian.com, techcrunch.com, cnet.com, and telegraph.co.uk. The search was carried out with pre-defined search terms on published news articles from January to October 2016. Technologies of interest were mHealth technologies, which have a potential impact on health care, regardless of their maturity level (i.e. under development, prototype, under control trial study, or pilot study).

Results: A total of 262 news articles were identified through electronic search and screened. After comparing against selection criteria, 75 mHealth innovative products/interventions were included in this review. Several wearable devices such as watches and wrist-worn bands were introduced for mental coaching, physical activity, fertility, fitness, sleep, blood pressure, and vital sign tracking. Numerous reports on smartphones connected appliances for medication adherence monitoring, baby tracking, woman health, including menstruation and pregnancy monitoring, were identified. Smartphone apps for body changes visualization, pre-hospital care, sleep screen, birth control, eye examination, rehabilitation, disease diagnosis and prescription, mental health services, and organ donation were also introduced.

Conclusion:This study demonstrated that personalized lifestyle and move towards consumer centered medicine monitoring through mobile health technologies such as wearable and connected appliances are emerging, which will be accessible for higher proportion of patients and health providers in the near future.

Keywords: mHealth; Innovation; Horizon Scanning
\end{abstract}

